\title{
Exosomes in the Diseased Brain: First Insights from In vivo Studies
}

\author{
Efrat Levy ${ }^{1,2 *}$ \\ ${ }^{1}$ Departments of Psychiatry, Biochemistry and Molecular Pharmacology, New York University Langone Medical Center, New \\ York, NY, USA, ${ }^{2}$ Center for Dementia Research, Nathan S. Kline Institute for Psychiatric Research, Orangeburg, NY, USA
}

Extracellular vesicles (EVs) are nanoscale size vesicles secreted by cells and are important mediators of intercellular communication and genetic exchange. Exosomes, EVs generated in endosomal multivesicular bodies, have been the focus of numerous publications as they have emerged as clinically valuable markers of disease states. Exosomes have been mostly studied from conditioned culture media and body fluids, with the difficulty of isolating exosomes from tissues having delayed their study in vivo. The implementation of a method designed to isolate exosomes from tissues, however, has yielded the first insights into characteristics of exosomes in the brain. It has been observed that brain exosomes from murine models of neurodegenerative diseases and human postmortem brains tend to mirror the protein content of the cells of origin,

OPEN ACCESS

Edited by:

Diana K. Sarko,

Southern Illinois University

Carbondale, USA

Reviewed by:

Varun Kesherwani,

University of Nebraska Medical

Center, USA

Felix Hernandez,

Universiad Autonoma de Madrid,

Spain

${ }^{*}$ Correspondence:

Efrat Levy

elevy@nki.rfmh.org

Specialty section: This article was submitted to

Neurodegeneration,

a section of the journal

Frontiers in Neuroscience

Received: 20 December 2016 Accepted: 07 March 2017

Published: 23 March 2017

Citation:

Levy E (2017) Exosomes in the Diseased Brain: First Insights from In vivo Studies.

Front. Neurosci. 11:142. doi: 10.3389/fnins.2017.00142 and interestingly, they are enriched with toxic proteins. Whether this enrichment with neurotoxic proteins is beneficial by relieving neurons of accumulated toxic material or detrimental to the brain by propagating pathogenicity throughout the brain remains to be answered. Here is summarized the first group of studies describing exosomes isolated from brain, results that demonstrate that exosomes in vivo reflect complex multicellular pathogenic processes in neurodegenerative disorders and the brain's response to injury and damage.

Keywords: extracellular vesicles, exosomes, brain, in vivo studies, neurodegeneration, neuroprotection

\section{INTRODUCTION}

Extracellular vesicles (EVs) are phospholipid bilayer membrane-enclosed vesicles that contain lipids, proteins, and RNA (mRNA and miRNA), secreted by cells into tissue extracellular spaces and biological fluids, and in vitro, into cultured conditioned media. in vitro studies have shown that cells release subpopulations of EVs with distinct molecular and biological properties (Lai et al., 2016; Willms et al., 2016), with the various species of EVs having different intracellular sites of origin (reviewed in van der Pol et al., 2012; Kowal et al., 2014; Kalra et al., 2016). Plasma membrane-derived microvesicles have a diameter of 100-1000 $\mathrm{nm}$. While these microvesicles are continuously shed from all viable cells, their release from the cell can be triggered under specific pathological conditions (Borroto-Escuela et al., 2015). The EVs species most extensively studied is the exosome, $20-100 \mathrm{~nm}$ vesicles formed by the invagination of the membrane of late endosome/multivesicular body (MVB) around cytoplasmic materials (reviewed in Kreimer et al., 2015; van der Pol et al., 2015). Mature exosomes remain inside the lumen of the MVB until they are secreted into the extracellular space when the MVB fuses with the plasma membrane (Stoorvogel et al., 2002). Secreted exosomes are taken-up by target cells, with the exosomal content delivered into the recipient cell (Fevrier and Raposo, 2004). Secreted exosomes were first isolated 
from the conditioned medium of immature sheep reticulocytes (Johnstone et al., 1987). Since then, in vitro studies have shown that exosomes are secreted by various cell types (Simpson et al., 2008), including neurons and astrocytes (Faure et al., 2006). Rat and mouse cortical neurons secrete exosomes in culture that have the typical features of size, density, and saponin sensitivity of exosomes secreted by other cells (Faure et al., 2006). Using proteomic methods, it was also shown that these exosomes resemble exosomes isolated from other non-neuronal cell types, containing typical exosomal markers such as Alix, flotillin, and tumor susceptibility gene-101 (TSG101), but also contain neuron-specific components (Faure et al., 2006).

Exosomes secretion was originally described as a complementary process to the lysosomal and proteosomal degradative pathways for shedding of obsolete membrane and cytosolic proteins (Johnstone et al., 1987). The identification of neuron-specific components associated with exosomes isolated from media conditioned by cultured cells (Faure et al., 2006) suggested that secreted exosomes have roles in cell signaling functions (Record et al., 2011) by shuttling cargo between cells and tissues (Smalheiser, 2007), contributing to the regulation of neurotransmitter receptor levels at the synapse, the production and turnover of myelin membranes proteins, and participating in the progression of neurodegenerative diseases (Gould et al., 2003; Vella et al., 2008; Izquierdo-Useros et al., 2011). A pathogenic function of exosomes has been additionally proposed, suggesting that exosomes can transfer pathogens between cells. One such pathogen that exploits this pathway is the prion protein $(\mathrm{PrP})$, the infectious particle responsible for transmissible neurodegenerative diseases such as CreutzfeldtJakob disease of humans and bovine spongiform encephalopathy of cattle (Vella et al., 2008). $\alpha$-synuclein, involved in the pathogenesis of Parkinson's disease is secreted in a calciumdependent manner by exosomes (Emmanouilidou et al., 2010). A pathogenic role for exosomes was also proposed for amyloid $\beta$ (A $\beta$ ) (Rajendran et al., 2006) and for phosphorylated tau (Saman et al., 2012), both deposited in the brain of Alzheimer's disease $(\mathrm{AD})$ patients. These data suggest that exosomes may contribute to intercellular membrane exchange and the spread of aggregation-prone proteins throughout the brain. However, exosomes may have a protective function by relieving the cells from toxic accumulation of peptides such as PrP, $\alpha$-synuclein, as well as $\mathrm{A} \beta$ and the amyloid $\beta$ precursor protein (APP) carboxyl terminal fragments (APP-CTFs) that accumulate intracellularly and disrupt the normal function of the endosomal-lysosomal system to degrade proteins. Thus, I have hypothesized that exosomes secretion plays a pleiotropic role in the brain, both beneficial to the cell by discarding accumulated, toxic material, but also potentially harmful to the brain by contributing to the extracellular build up and transport between cells of toxic material.

Isolating exosomes from in vitro sources has provided data on the level and content of exosomes secretion by specific cell types but cannot provide information on the in vivo physiological effect on exosomes secretion by multiple cell types under normal, developmental as well as pathological conditions. While exosomes isolated from cerebrospinal fluid (CSF) are enriched in proteins derived from brain, including neuronal and microglia markers, the origin of exosomes in the CSF is not strictly from the brain (reviewed in Vella et al., 2016). Although CSF-derived exosomes can serve as biomarkers for brain disease when changes in specific proteins, lipids, or RNA are detected, there is a need to investigate the changes that occur in the brain environment leading to disease initiation and progression. Moreover, given that in most neurodegenerative disorders the primary changes occur in specific localization within the brain, followed by propagation of pathology into welldefined brain regions, it is likely that the levels of secretion and cargo compositions of exosomes are not homogeneous among various brain regions. Understanding this diversity requires the investigation of exosomes content not only in the brain as a whole but also in distinct brain regions.

Understanding exosomes-based signaling in the brain will likely advance knowledge of neurobiology in the non-affected brain, under normal conditions, in addition to understanding disease-related roles of exosomes. The first protocol to isolate exosomes from brain tissues was developed by Perez-Gonzalez et al. (2012), and a second method has been described, a protein organic solvent precipitation method (Gallart-Palau et al., 2016). Both methods efficiently isolate brain tissue exosomes in amounts sufficient to characterize exosomes by multiple technologies, including "omics" (Gallart-Palau et al., 2016 and Perez-Gonzalez and Levy, personal communication). Given the recent development of such methodology, the number of publications of studies describing brain exosomes is limited. Nevertheless, these studies highlight the unique data that can be obtained by directly isolating and characterizing brain exosomes. This review describes the data obtained from exosomes isolated from brain tissue of patients with neurodegenerative disorders, data that could not be obtained from exosomes isolated from body fluids or conditioned media.

\section{METHOD TO ISOLATE EXOSOMES FROM BRAIN TISSUE}

While exosomes have been successfully isolated from cell culture media and body fluids including CSF, blood, saliva, and urine (Lakkaraju and Rodriguez-Boulan, 2008; Simpson et al., 2008), similar isolation methods for brain exosomes yielded vesicles contaminated with cellular debris, as observed by electron microscopy and Western blot analysis with antibodies to intracellular compartments. We have successfully isolated exosomes-enriched EVs from brain that are not contaminated by cellular debris or intracellular vesicles (Perez-Gonzalez et al., 2012). While this protocol requires rigor and precise performance and we have found that deviation from some of the technique's details can prevent success, several researchers have successfully isolated brain exosomes following our published protocol; their findings are described below. This protocol starts with a gentle dissociation of the tissue to free the brain extracellular space, keeping the cells intact, the same method we have been using for the isolation of intact neurons, glia, smooth muscle cells, and endothelial cells from brain for culture 
(Jung and Levy, 2005). The brain tissue is not homogenized, but gently disassociated. Unfortunately, in some manuscripts the method has been described as a "homogenization" although the actual technique described involved the gentle dissociation as described in Perez-Gonzalez et al. (2012). After a low-speed centrifugation to discard large debris, the sample is sequentially filtered through 0.4 and $0.2 \mu \mathrm{m}$ filters to further eliminate remaining debris and vesicles larger than $200 \mathrm{~nm}$, followed by low-speed centrifugations and ultracentrifugations (see Figure 1 and Perez-Gonzalez et al., 2012 for details of our protocol). Electron microscopy of brain EVs demonstrated that this method yields EVs preparations free of debris and subcellular organelles, such as the nucleus, endoplasmic reticulum, mitochondria, and Golgi (Perez-Gonzalez et al., 2012). Western blot analysis demonstrated that the EVs did not contain proteins of intracellular compartments such as endoplasmic reticulum or Golgi, but contained markers of exosomes, indicating the purity of the samples. This procedure yields vesicles that match in shape, size, density, and protein content exosomes previously isolated from body fluids or from the conditioned media of various types of cell cultures (Thery et al., 2006; Simpson et al., 2008). As was previously shown for human urine (Zhou et al., 2006), CSF (Harrington et al., 2009), and plasma (Grant et al., 2011), intact exosomes can be isolated from frozen brain tissues as well. Electron microscopy revealed no difference between exosomes isolated from human brain tissues frozen for several years at $-80^{\circ} \mathrm{C}$, mouse brains frozen for long periods of time at $-80^{\circ} \mathrm{C}$, and freshly isolated mouse brains.

Each of the studies that have used this isolation method, described below, employed Western blotting and immuno-EM with exosomes-specific antibodies to identify exosomes within the isolated EVs. These include antibodies to proteins such as the TSG101, required for endosomal sorting complex required for transport (ESCRT) recruitment to endosomal membranes during the process of exosomes generation (de Gassart et al., 2004), multiple rab GTPase, key regulators of intracellular membrane trafficking, including rab27a and rab27b that function in MVB docking at the plasma membrane (Ostrowski et al., 2010), and rab35, which regulates exosomes secretion (Hsu et al., 2010). The endosomal origin of exosomes is supported by the identification of rab5 isoforms (rab5a, rab5b, rab5c), proteins essential for the fusion of early endosomes and regulation of endocytosis, in exosomes (Agola et al., 2011; Pfeffer, 2013). Brain exosomes also contained various tetraspanin proteins, such as CD9, CD63, CD81, abundantly present in the endosomal-lysosomal pathway where they are enriched on the intraluminal vesicles and extensively used as markers of exosomes (Abache et al., 2007; Pols and Klumperman, 2009; Jorgensen et al., 2013).

\section{EXOSOMES ISOLATED FROM BRAIN PROVIDE UNIQUE DATA ON THE ROLE OF EXOSOMES IN NEURODEGENERATIVE DISORDERS}

Multiple studies have shown that the levels and composition of exosomes are affected by, and contribute to, disease progression (Kreimer et al., 2015) although the mechanisms are poorly understood (Urbanelli et al., 2013). Changes in exosomes generation, secretion, uptake, or clearance, manifested by changes in the number of exosomes in the brain extracellular space, may have either protective or pathogenic effect. Given that exosomes contain amyloidogenic and toxic material, exosomes release can serve a neuroprotective function through the elimination of neurotoxic molecules that cause neuronal endosomal-autophagic-lysosomal abnormalities and functional sequelae, including memory loss. Recent studies have shown that exosomes uptake by microglia is more efficient than by neurons (Fitzner et al., 2011; Yuyama et al., 2012), suggesting that exosomes release may have a neuroprotective function by allowing a neuron to shed deleterious components that are then targeted to and degraded by non-neuronal cells (Joshi et al., 2015). Unfortunately, the elimination of cytotoxic material associated with exosomes by neurons, if not efficiently degraded, may lead to a propagation of toxicity and pathology in the brain, as has been suggested for prion diseases (Vella et al., 2008). The direct isolation of exosomes from the tissue has the advantage of revealing in vivo processes in the normal brain as well as changes that occur within the environment of the brain during normal aging or those leading to disease. While regional analyses can be performed, such studies provide limited information on the cell of origin of the exosomes or the mechanisms leading to altered exosomes levels in the brain. To this end, in vivo studies often need to be accompanied by in vitro studies in which exosomes are isolated from specific cell types, such as neurons, astrocytes, and microglia. This section provides published examples of the unique data obtained from exosomes isolated from brain tissue. While the extent of published studies to date remains limited, these studies and their findings encourage the use of this

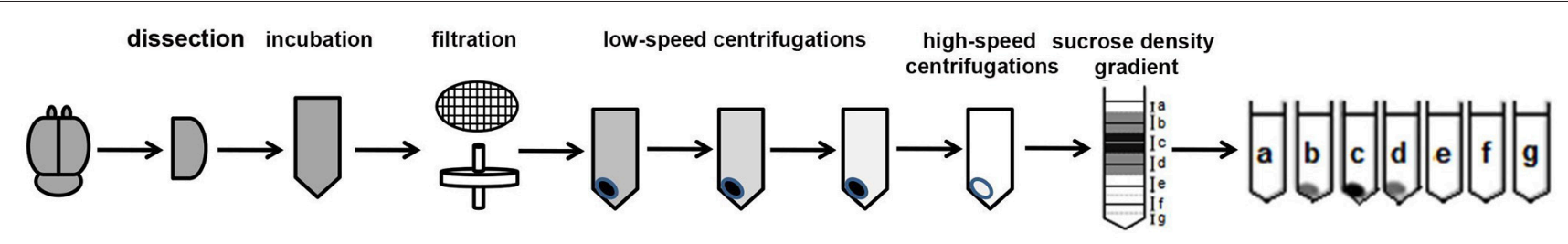

FIGURE 1 | Brain exosome isolation experimental flow chart. The steps of the experimental procedure designed to isolate and purify brain exosomes are described. 
methodology to further delineate the roles of exosomes secretion, uptake, and signaling in the normal and diseased brain.

\section{The Normal Brain}

Isolation of exosomes from brain can serve as a confirmation that processes observed in the in vitro model also occur in vivo in the brain. For example, one study identified a novel mechanism that regulates the activity of the membranebound receptor for platelet-derived growth factor A (PDGFR $\alpha$ ), crucial for controlling the production of oligodendrocytes for myelination (Pituch et al., 2015). This study tested whether galactosylceramides (sulfatides) contribute to the regulation of oligodendrogenesis by modulating PDGFR $\alpha$ function. It was found that increased sulfatides levels in multipotential neural precursors lead to a reduced production of oligodendrocytes progenitor cells and oligodendrocytes. The activity of PDGFR $\alpha$ depends on its localization to detergent-resistant membrane domains in the plasma membrane and interaction with integrin complexes and therefore, the diminished association of PDGFR $\alpha$ with these domains due to increased sulfatides levels resulted in exacerbated PDGFR $\alpha$ activity. This study identified a novel mechanism that regulates the secretion of PDGFR $\alpha$ in multipotential neural precursor cells, in glial cells, and in the brain cortex via exosomal shedding. While the in vitro data has shown that PDGFR $\alpha$ is secreted by multipotential neural precursors and by glial cells via exosomes, the in vivo study has shown that PDGFR $\alpha$ is secreted via exosomes during the peak of myelination (Pituch et al., 2015). Exosomal PDGFR $\alpha$ secretion may be a physiologically relevant function of exosomal secretion by which oligodendrocytes progenitor cells rapidly and locally regulate the concentration of the receptor. This can have an impact on the receptor-mediated responses to proliferation, survival and differentiation. Thus, the in vivo studies provide further evidence that exosomal regulation of PDGFR $\alpha$ is part of a larger regulatory program involved in myelination.

\section{Alzheimer's Disease}

Extensive research has shown that several metabolites of the APP are toxic to neuronal cells. A central pathological feature of $\mathrm{AD}$ is the accumulation of $\mathrm{A} \beta$ in the brain, and it was suggested that $A \beta$ has an important role in the pathogenesis of neuronal dysfunction in the disease (reviewed in Selkoe, 1991; Hardy and Selkoe, 2002; Stine et al., 2003). Recent in vitro and in vivo studies show that other APP metabolites, mainly the $\beta$-secretase derived APP-CTFs, are neurotoxic and can cause memory loss (Jiang et al., 2010; Deyts et al., 2012; Tamayev and D’Adamio, 2012; Tamayev et al., 2012). Most significantly, it was shown that APP- $\beta$ CTF cause neuronal endosomal-lysosomal abnormalities and resulting memory loss (Neve et al., 1996; Oster-Granite et al., 1996; Neve and Robakis, 1998; McPhie et al., 2001; Jiang et al., 2010; Deyts et al., 2012; Flammang et al., 2012; Lauritzen et al., 2012, 2016; Oules et al., 2012; Tamayev and D'Adamio, 2012; Tamayev et al., 2012). Exosomal proteins were found to accumulate in the amyloid plaques in the brain of $\mathrm{AD}$ patients (Rajendran et al., 2006) and therefore it was proposed that exosomes participate in the pathogenesis of $\mathrm{AD}$. Exosomes isolated from the conditioned medium of neuronal cell cultures contain as cargo the full-length APP, APP metabolites, and key enzymes that cleave both full-length APP and CTFs, and exosomes are one site where APP processing occurs (Rajendran et al., 2006; Vingtdeux et al., 2007; Escrevente et al., 2008; Sharples et al., 2008). The presence of precursors to amyloidogenic proteins and amyloidogenic proteins within exosomes suggest that exosomes can release amyloidogenic material and transmit it between cells, playing a pathogenic role in neurodegenerative diseases.

Isolation of exosomes from mouse brains had shown that murine brain exosomes contain full-length APP and the APP metabolites APP-CTFs and A $\beta$ (Perez-Gonzalez et al., 2012) as was shown previously for exosomes isolated from the media cultured by neuronal cells in vitro (Vingtdeux et al., 2007). A novel finding was that exosomes isolated from brains of mice are enriched with APP-CTFs compared to brain homogenates (Perez-Gonzalez et al., 2012). These results imply the existence of a set of mechanisms driving an enrichment of APP-CTFs in exosomes. Additionally brain exosomes were isolated from brains of transgenic mice overexpressing human APP with the K670N/M671L Swedish double mutation (Tg2576; Hsiao et al., 1996). The findings showed that the amount of flAPP and APPCTFs secreted out of the cell in brain exosomes is proportional to their expression levels in the brain, but that the exosomes are greatly enriched with APP-CTFs (Perez-Gonzalez et al., 2012). Immuno-EM using antibodies directed to cytoplasmic or extracellular epitopes of APP revealed that the exosomes are oriented with the cytoplasmic-side facing inward (Pisitkun et al., 2004). Therefore, cleavage of APP results in capture of APPCTFs inside exosomes. Thus, the enrichment of APP-CTFs in exosomes isolated from brains results from endosomal APPCTFs that were captured and carried by exosomes combined with metabolism of flAPP in the exosomes. Contrary to flAPP and APP-CTFs, only a minute fraction of total $A \beta(<1 \%)$ is associated with exosomes (Rajendran et al., 2006). The orientation of APP in the membrane of exosomes would result in the secretion of exosomal-generated $A \beta$ into the extracellular space, not into the vesicles. Data suggest that $A \beta$ binds to exosomes that possibly act as a seed for plaque formation (reviewed in Dinkins et al., 2016a).

Isolation of exosomes from murine brain also enables direct in vivo study of the effect of exosomes release on amyloidogenesis. This was studied in a transgenic mouse expressing five familial mutations in the human APP and presenilin 1 genes (5XFAD). This model primarily produces $\mathrm{A} \beta 42$, with amyloid plaques forming as early as at 3 months of age (Oakley et al., 2006). Prevention of exosomes secretion by the inhibition of neutral sphingomyelinase 2 (nSMase2), a key regulatory enzyme generating ceramide from sphingomyelin, with GW4869 in the 5XFAD transgenic mouse reduced total $A \beta 42$ levels and plaque load in the brain (Dinkins et al., 2014). In genetically nSMase2-deficient 5XFAD mice, reduced glial activation, total $\mathrm{A} \beta 42$ and plaque burden, and tau phosphorylation, along with an improvement in a fearconditioned learning task were observed (Dinkins et al., 2016b). These findings are consistent with the idea that APP metabolite loaded exosomes may, under some conditions, contribute to amyloidogenesis. Additionally, the isolation of exosomes from 
mouse brain facilitated the identification of a difference between females and males, showing that the reduction of total $A \beta 42$ levels and the number of plaques in the brain with a reduction in nSMase2 function was significantly greater in male than female mice (Dinkins et al., 2014), suggesting sex differences in brain exosome secretion or function. This information may provide a mechanism for the higher vulnerability of females to $\mathrm{AD}$ pathogenesis: sex differences have been reported in $\mathrm{AD}$, with evidence that women expressing the apolipoprotein $\mathrm{E} 4$ allele have a higher risk of being affected by $\mathrm{AD}$ than men, regardless of longevity and other disease mortality factors (Payami et al., 1994; Buckwalter et al., 1996; Farrer et al., 1997; Andersen et al., 1999; Bretsky et al., 1999; Lobo et al., 2000; Mortensen and Hogh, 2001; Brookmeyer et al., 2002; Barnes et al., 2003; Baum, 2005; Beydoun et al., 2010; Altmann et al., 2014).

Another major hallmark of $\mathrm{AD}$ is the accumulation of pathological tau protein, spreading in well-defined stages between different regions of the brain, potentially via exosomes. Exosomes-associated tau phosphorylated at Thr-181 (AT270), an established phosphorylated tau biomarker for $\mathrm{AD}$, was found in tissue culture media and in human CSF samples, suggesting that exosomes-mediated secretion of phosphorylated tau plays a role in the abnormal processing of tau in early AD (Saman et al., 2012). It was hypothesized that microglia, the primary phagocytes in the brain, facilitate tau protein propagation between neurons by phagocytosing and exocytosing tau, supported by the finding that depleting microglia dramatically suppressed the propagation of tau (Asai et al., 2015). An in vitro study had shown that microglia are more efficient at the uptake of tau as compared to neurons and astrocytes. Isolation of exosomes from the brain of a mouse model of tau propagation revealed that vesicles that contain exosomal proteins contain tau and that exosomes isolated from the brain of these mice transduce tau into primary cultured neurons (Asai et al., 2015). Moreover, microglial depletion partially reduced tau content in exosomes, and inhibiting exosomes synthesis significantly reduced tau propagation in vitro and in vivo. These data suggest that exosomes may play a prominent role in the progression of tauopathy within the brain (Asai et al., 2015).

A study that isolated exosomes from the brain of a tau transgenic mouse that expresses four-repeat tau with the P301L mutation showed that exosomes levels of tau were significantly higher in transgenic mice that have pronounced tau pathology (Polanco et al., 2016). Tau in the vesicles was differentially phosphorylated, although to a lower degree than in the brain cells from which they were derived. Several phospho-epitopes (AT8, AT100, and AT180) thought to be critical for tau pathology were undetected in brain exosomes. Despite this, exosomes derived from transgenic mice were capable of seeding tau aggregation in a threshold-dependent manner, demonstrating that taucontaining exosomes can transmit tau pathology. Moreover, the characteristics of tau in the exosomes and the high seeding threshold identified may explain why tau pathology develops very slowly in neurodegenerative diseases such as AD (Polanco et al., 2016). In another study, the seeding potential of lysates and EVs enriched for exosomes from wild-type and the tau transgenic mouse brains was compared, showing that the transgenic exosomes cause increased tau phosphorylation and soluble oligomer formation in a manner comparable to that of freely available proteins in brain lysates, a prerequisite for the formation of mature protein aggregate (Baker et al., 2016). These in vivo studies show that exosomes can propagate tau pathology, can carry tau seeds able to induce tau aggregation in recipient cells, and that such tau is phosphorylated at epitopes found in $\mathrm{AD}$ patients.

\section{Dementia with Lewy Body and Parkinson's Disease}

$\alpha$-synuclein aggregation plays a central role in Parkinson's disease pathology. Exosomes released from $\alpha$-synuclein overexpressing $\mathrm{SH}-\mathrm{SY} 5 \mathrm{Y}$ cells contain $\alpha$-synuclein, and these exosomes were capable of efficiently transferring $\alpha$-synuclein to normal $\mathrm{SH}$ SY5Y cells (Emmanouilidou et al., 2010; Alvarez-Erviti et al., 2011). An in vitro study that examined the role of the ESCRT pathway in the propagation of $\alpha$-synuclein has shown that disruption of the ESCRT transport system resulted in increased exocytosis of $\alpha$-synuclein (Spencer et al., 2016). The in vitro study also showed that the ESCRT protein-charged multivesicular body protein (CHMP2B) affects the release of $\alpha$-synuclein, and downregulation of CHMP2B affects the intracellular capacity of cells to clear $\alpha$-synuclein, leading to greater release of $\alpha$ synuclein into the media. Consistent with these findings, lower levels of CHMP2B were found in the brain of patients diagnosed with dementia with Lewy body as compared with controls, thus generating a roadblock of endocytosed $\alpha$-synuclein. In order to determine whether $\alpha$-synuclein is released via exosomes in vivo, exosomes were isolated from brain of patients with dementia with Lewy body, revealing that the exosomes contained $\alpha$-synuclein, while exosomes isolated from control individuals did not (Spencer et al., 2016). These data suggest that neurons compensate for dysfunction of the endosomal-lysosomal pathway that cause accumulation of material such as $\alpha$-synuclein in MVB by enhanced release of exosomes, potentially increasing cell-to-cell propagation of $\alpha$-synuclein in neurological disorders with parkinsonism, including Parkinson's disease and dementia with Lewy body.

\section{Amyotrophic Lateral Sclerosis and Frontotemporal Lobar Degeneration}

Similar to dementia with Lewy body, data on exosomesassociated TDP-43, a pathological hallmark of amyotrophic lateral sclerosis and frontotemporal lobar degeneration, suggest that enhanced exosomes release is beneficial to neuronal survival, in spite of potential increase in disease propagation (Iguchi et al., 2016). in vitro investigation of the role of exosomes in the secretion and propagation of TDP-43 aggregates have shown that TDP-43 is secreted by neurons but not by astrocytes or microglia in association with exosomes. Isolation of exosomes from human brains had shown that levels of exosomal TDP-43 full length and C-terminal fragment species are upregulated in human amyotrophic lateral sclerosis brains. When Neuro2a cells were exposed to exosomes from amyotrophic lateral sclerosis brain, but not from control brain, cytoplasmic redistribution of TDP-43 was observed (Iguchi et al., 2016). These data suggest that secreted exosomes contribute to propagation of TDP43 proteinopathy. However, inhibition of exosomes secretion 
provoked formation of TDP-43 aggregates in Neuro2a cells and exacerbated TDP-43 neuronal aggregation. Moreover, the disease phenotypes of transgenic mice expressing human TDP43A315T mutant was exacerbated following in vivo inhibition of exosomes secretion. Given that cytoplasmic TDP-43 aggregation occurs in amyotrophic lateral sclerosis and frontotemporal lobar degeneration, these results suggest that exosomes containing pathological TDP-43 play a role in the propagation of TDP43 proteinopathy. Thus, a therapeutic strategy for amyotrophic lateral sclerosis based on inhibition of exosomes production based on in vivo data that suggest that exosomes secretion plays an overall beneficial role in neuronal clearance of pathological TDP-43 would be inadvisable (Iguchi et al., 2016).

\section{Simian Immunodeficiency Virus-Induced Brain Disease}

Investigation of the role of exosomes in simian immunodeficiency virus (SIV)-induced brain disease was conducted by the isolation and characterization of exosomes from the brains of rhesus macaques. Small RNA sequencing revealed higher miR-21 levels in exosomes from SIV encephalitic brains compared with controls (Yelamanchili et al., 2015a,b). In situ hybridization revealed increased miR-21 expression in neurons and macrophage/microglial cells/nodules during SIV induced brain disease. in vitro culture of macrophages have shown that miR-21 is released via exosomes and is neurotoxic via activation of TLR7 when compared to exosomes derived from miR-21 knockout mice (Yelamanchili et al., 2015a,b). This study further emphasizes the value of the characterization of exosomal response in the affected brain in combination with an in vitro study to further define the mechanism of action.

\section{Traumatic Brain Injury}

The same exosomes-associated miRNA involved in SIV-induced brain disease was found to have a role in traumatic brain injury (TBI; Harrison et al., 2016). While several experiments have shown the effects of exosomes-associated miRNA on cells in culture, this study investigated how miRNA content of exosomes is altered in vivo in the brain. Exosomes were isolated from the brain of a mouse model of TBI (controlled cortical impact) and controls. Profiling of miRNA in the exosomes has shown changes in miRNA species, with miR-21 showing the largest change between conditions. Highlighting the value of isolating exosomes from the brain directly, the miRNA profiles found in this study were not identical to those seen in exosomes isolated from human CSF after TBI described in a previous study (Patz et al., 2013). It remains to be determined whether the difference between the studies is due solely to a difference between CSF and brain exosomes, or due to a difference between TBI in humans and the mouse model, critical to the interpretation of further studies. Study of the mouse brain has shown that the expression of miR21 in the brain was primarily localized to neurons, not microglia, near the lesion site, while adjacent to these miR-21-expressing neurons were activated microglia. The concurrent elevation of miR-21 in neurons with the increase in miR-21 in exosomes suggests that miR-21 secreted from neurons in association with exosomes is a potential mechanism of cell-to-cell communication resulting in microglia activation as a protective mechanism. Thus, while a neurotoxic function of exosomes-associated miR21 was shown in the brain of SIV-induced rhesus macaques brain disease (Yelamanchili et al., 2015a,b), a protective function is suggested, but needs to be proven, in a mouse model of TBI (Harrison et al., 2016).

\section{Schizophrenia and Bipolar Disorder}

In another study, well-preserved exosomal miRNAs were isolated from frozen postmortem prefrontal cortices and submitted to RNA profiling to examine whether they reflect disease-specific changes in psychiatric disorders (Banigan et al., 2013). The expression of certain exosomal miRNA in the brains of patients diagnosed with schizophrenia and bipolar disorder differed from controls, reflecting either disease-specific or common aberrations in these patients. RT-PCR validation confirmed that two miRNAs, miR-497 in schizophrenia brain samples and miR29c in bipolar disorder brain samples, have significantly higher expression when compared to control samples (Banigan et al., 2013). These results show that brain exosomes characterization has the potential to identify the cellular mechanism of disease pathology, as well as showing the potential of exosomes-derived miRNAs to serve as biomarkers for various disorders.

\section{CONCLUSIONS}

Studies of exosomes isolated from brain tissue demonstrate that unique, brain-specific data can be obtained, findings that are not available from exosomes isolated from conditioned media or body fluids. Moreover, they show that exosomes released into the extracellular space of human and mouse brains under disease conditions have different exosomal cargo proteins and miRNAs compared with unaffected controls. However, these in vivo studies are not able to identify specific cellular sources of these exosomes as well as cell biological and vesicle trafficking alterations that alter exosomes, data that can be obtained from cultured cells. Given that brain-derived exosomes can reflect disease processes, they may also be used to evaluate the effect of therapeutic interventions on specific markers.

As the field continues to undertake a comprehensive and integrated analysis of brain tissue exosomes, exosomes secreted from the various cell-types found within the brain, and from the CSF, our understanding of exosomes secretion as a cellular rescue pathway and as a potential mechanisms for disease propagation will grow. Additionally, the newly appreciated value of exosomes as biomarkers for disease diagnosis and informers of pathogenic processes within cells will likely lead to greater use of exosomal analyses as translation research and clinical tools.

\section{AUTHOR CONTRIBUTIONS}

EL wrote the review.

\section{FUNDING}

This work was supported by the National Institutes of Health (AG017617 and AG037693). 


\section{REFERENCES}

Abache, T., Le Naour, F., Planchon, S., Harper, F., Boucheix, C., and Rubinstein, E. (2007). The transferrin receptor and the tetraspanin web molecules CD9, CD81, and CD9P-1 are differentially sorted into exosomes after TPA treatment of K562 cells. J. Cell. Biochem. 102, 650-664. doi: 10.1002/jcb.21318

Agola, J. O., Jim, P. A., Ward, H. H., Basuray, S., and Wandinger-Ness, A. (2011). Rab GTPases as regulators of endocytosis, targets of disease and therapeutic opportunities. Clin. Genet. 80, 305-318. doi: 10.1111/j.1399-0004.2011.01724.x

Altmann, A., Tian, L., Henderson, V. W., Greicius, M. D., and the Alzheimer's Disease Neuroimaging Initiative Investigators (2014). Sex modifies the APOErelated risk of developing Alzheimer disease. Ann. Neurol. 75, 563-573. doi: 10.1002/ana.24135

Alvarez-Erviti, L., Seow, Y., Schapira, A. H., Gardiner, C., Sargent, I. L., Wood, M. J., et al. (2011). Lysosomal dysfunction increases exosomemediated $\alpha$-synuclein release and transmission. Neurobiol. Dis. 42, 360-367. doi: 10.1016/j.nbd.2011.01.029

Andersen, K., Launer, L. J., Dewey, M. E., Letenneur, L., Ott, A., Copeland, J. R., et al. (1999). Gender differences in the incidence of AD and vascular dementia: the EURODEM Studies. EURODEM Incidence Research Group. Neurology 53, 1992-1997. doi: 10.1212/wnl.53.9.1992

Asai, H., Ikezu, S., Tsunoda, S., Medalla, M., Luebke, J., Haydar, T., et al. (2015). Depletion of microglia and inhibition of exosome synthesis halt tau propagation. Nat. Neurosci. 18, 1584-1593. doi: 10.1038/nn.4132

Baker, S., Polanco, J. C., and Gotz, J. (2016). Extracellular vesicles containing P301L mutant tau accelerate pathological tau phosphorylation and oligomer formation but do not seed mature neurofibrillary tangles in ALZ17 mice. J. Alzheimers Dis. 54, 1207-1217. doi: 10.3233/JAD-160371

Banigan, M. G., Kao, P. F., Kozubek, J. A., Winslow, A. R., Medina, J., Costa, J., et al. (2013). Differential expression of exosomal microRNAs in prefrontal cortices of schizophrenia and bipolar disorder patients. PLOS ONE 8:e48814. doi: 10.1371/journal.pone.0048814

Barnes, L. L., Wilson, R. S., Schneider, J. A., Bienias, J. L., Evans, D. A., and Bennett, D. A. (2003). Gender, cognitive decline, and risk of AD in older persons. Neurology 60, 1777-1781. doi: 10.1212/01.WNL.0000065892.67099.2A

Baum, L. W. (2005). Sex, hormones, and Alzheimer's disease. J. Gerontol. A Biol. Sci. Med. Sci. 60, 736-743. doi: 10.1093/gerona/60.6.736

Beydoun, M. A., Boueiz, A., Abougergi, M. S., Kitner-Triolo, M. H., Beydoun, H. A., Resnick, S. M., et al. (2010). Sex differences in the association of the apolipoprotein E $\varepsilon 4$ allele with incidence of dementia, cognitive impairment, and decline. Neurobiol. Aging 33, 720e4-731.e4. doi: 10.1016/j.neurobiolaging.2010.05.017

Borroto-Escuela, D. O., Agnati, L. F., Bechter, K., Jansson, A., Tarakanov, A. O., and Fuxe, K. (2015). The role of transmitter diffusion and flow versus extracellular vesicles in volume transmission in the brain neural-glial networks. Philos. Trans. R. Soc. Lond. B. Biol. Sci. 370:20140183. doi: 10.1098/rstb.2014.0183

Bretsky, P. M., Buckwalter, J. G., Seeman, T. E., Miller, C. A., Poirier, J., Schellenberg, G. D., et al. (1999). Evidence for an interaction between apolipoprotein E genotype, gender, and Alzheimer disease. Alzheimer Dis. Assoc. Disord. 13, 216-221. doi: 10.1097/00002093-199910000-00007

Brookmeyer, R., Corrada, M. M., Curriero, F. C., and Kawas, C. (2002). Survival following a diagnosis of Alzheimer disease. Arch. Neurol. 59, 1764-1767. doi: 10.1001/archneur.59.11.1764

Buckwalter, J. G., Rizzo, A. A., McCleary, R., Shankle, R., Dick, M., and Henderson, V. W. (1996). Gender comparisons of cognitive performances among vascular dementia, Alzheimer disease, and older adults without dementia. Arch. Neurol. 53, 436-439. doi: 10.1001/archneur.1996.00550050066025

de Gassart, A., Geminard, C., Hoekstra, D., and Vidal, M. (2004). Exosome secretion: the art of reutilizing nonrecycled proteins? Traffic 5, 896-903. doi: 10.1111/j.1600-0854.2004.00223.x

Deyts, C., Vetrivel, K. S., Das, S., Shepherd, Y. M., Dupre, D. J., Thinakaran, G., et al. (2012). Novel GalphaS-protein signaling associated with membranetethered amyloid precursor protein intracellular domain. J. Neurosci. 32, 1714-1729. doi: 10.1523/JNEUROSCI.5433-11.2012

Dinkins, M. B., Dasgupta, S., Wang, G., Zhu, G., and Bieberich, E. (2014). Exosome reduction in vivo is associated with lower amyloid plaque load in the 5XFAD mouse model of Alzheimer's disease. Neurobiol. Aging 35, 1792-1800. doi: 10.1016/j.neurobiolaging.2014.02.012
Dinkins, M. B., Enasko, J., Hernandez, C., Wang, G., Kong, J., Helwa, I., et al. (2016b). Neutral sphingomyelinase-2 deficiency ameliorates Alzheimer's disease pathology and improves cognition in the 5XFAD mouse. J. Neurosci. 36, 8653-8667. doi: 10.1523/JNEUROSCI.1429-16.2016

Dinkins, M. B., Wang, G., and Bieberich, E. (2016a). Sphingolipid-enriched extracellular vesicles and Alzheimer's disease: a decade of research. J. Alzheimers Dis. doi: 10.3233/JAD-160567. [Epub ahead of print].

Emmanouilidou, E., Melachroinou, K., Roumeliotis, T., Garbis, S. D., Ntzouni, M., Margaritis, L. H., et al. (2010). Cell-produced $\alpha$-synuclein is secreted in a calcium-dependent manner by exosomes and impacts neuronal survival. $J$. Neurosci. 30, 6838-6851. doi: 10.1523/JNEUROSCI.5699-09.2010

Escrevente, C., Morais, V. A., Keller, S., Soares, C. M., Altevogt, P., and Costa, J. (2008). Functional role of N-glycosylation from ADAM10 in processing, localization and activity of the enzyme. Biochim. Biophys. Acta 1780, 905-913. doi: 10.1016/j.bbagen.2008.03.004

Farrer, L. A., Cupples, L. A., Haines, J. L., Hyman, B., Kukull, W. A., Mayeux, R., et al. (1997). Effects of age, sex, and ethnicity on the association between apolipoprotein E genotype and Alzheimer disease. A meta-analysis. APOE and Alzheimer disease meta-analysis consortium. JAMA 278, 1349-1356. doi: 10.1001/jama.1997.03550160069041

Faure, J., Lachenal, G., Court, M., Hirrlinger, J., Chatellard-Causse, C., Blot, B., et al. (2006). Exosomes are released by cultured cortical neurones. Mol. Cell. Neurosci. 31, 642-648. doi: 10.1016/j.mcn.2005.12.003

Fevrier, B., and Raposo, G. (2004). Exosomes: endosomal-derived vesicles shipping extracellular messages. Curr. Opin. Cell Biol. 16, 415-421. doi: 10.1016/j.ceb. 2004.06.003

Fitzner, D., Schnaars, M., van Rossum, D., Krishnamoorthy, G., Dibaj, P., Bakhti, M., et al. (2011). Selective transfer of exosomes from oligodendrocytes to microglia by macropinocytosis. J. Cell Sci. 124(Pt 3), 447-458. doi: 10.1242/jcs. 074088

Flammang, B., Pardossi-Piquard, R., Sevalle, J., Debayle, D., Dabert-Gay, A. S., Thevenet, A., et al. (2012). Evidence that the amyloid- $\beta$ protein precursor intracellular domain, AICD, derives from $\beta$-secretase-generated C-terminal fragment. J. Alzheimers Dis. 30, 145-153. doi: 10.3233/JAD-2012-112186

Gallart-Palau, X., Serra, A., and Sze, S. K. (2016). Enrichment of extracellular vesicles from tissues of the central nervous system by PROSPR. Mol. Neurodegener. 11, 41. doi: 10.1186/s13024-016-0108-1

Gould, S. J., Booth, A. M., and Hildreth, J. E. (2003). The Trojan exosome hypothesis. Proc. Natl. Acad. Sci. U.S.A. 100, 10592-10597. doi: 10.1073/pnas. 1831413100

Grant, R., Ansa-Addo, E., Stratton, D., Antwi-Baffour, S., Jorfi, S., Kholia, S., et al. (2011). A filtration-based protocol to isolate human plasma membrane-derived vesicles and exosomes from blood plasma. J. Immunol. Methods 371, 143-151. doi: 10.1016/j.jim.2011.06.024

Hardy, J., and Selkoe, D. J. (2002). The amyloid hypothesis of Alzheimer's disease: progress and problems on the road to therapeutics. Science 297, 353-356. doi: 10.1126/science.1072994

Harrington, M. G., Fonteh, A. N., Oborina, E., Liao, P., Cowan, R. P., McComb, G., et al. (2009). The morphology and biochemistry of nanostructures provide evidence for synthesis and signaling functions in human cerebrospinal fluid. Cerebrospinal Fluid Res. 6:10. doi: 10.1186/1743-8454-6-10

Harrison, E. B., Hochfelder, C. G., Lamberty, B. G., Meays, B. M., Morsey, B. M., Kelso, M. L., et al. (2016). Traumatic brain injury increases levels of miR-21 in extracellular vesicles: implications for neuroinflammation. FEBS Open Bio 6, 835-846. doi: 10.1002/2211-5463.12092

Hsiao, K., Chapman, P., Nilsen, S., Eckman, C., Harigaya, Y., Younkin, S., et al. (1996). Correlative memory deficits, $A \beta$ elevation and amyloid plaques in transgenic mice. Science 274, 99-102. doi: 10.1126/science.274.5284.99

Hsu, C., Morohashi, Y., Yoshimura, S., Manrique-Hoyos, N., Jung, S., Lauterbach, M. A., et al. (2010). Regulation of exosome secretion by Rab35 and its GTPase-activating proteins TBC1D10A-C. J. Cell Biol. 189, 223-232. doi: $10.1083 /$ jcb.200911018

Iguchi, Y., Eid, L., Parent, M., Soucy, G., Bareil, C., Riku, Y., et al. (2016). Exosome secretion is a key pathway for clearance of pathological TDP-43. Brain 139(Pt 12), 3187-3201. doi: 10.1093/brain/aww237

Izquierdo-Useros, N., Naranjo-Gomez, M., Erkizia, I., Puertas, M. C., Borras, F. E., Blanco, J., et al. (2011). HIV and mature dendritic cells: trojan exosomes riding the Trojan horse? PLoS Pathog. 6:e1000740. doi: 10.1371/journal.ppat.1000740 
Jiang, Y., Mullaney, K. A., Peterhoff, C. M., Che, S., Schmidt, S. D., Boyer-Boiteau, A., et al. (2010). Alzheimer's-related endosome dysfunction in Down syndrome is $\mathrm{A} \beta$-independent but requires APP and is reversed by BACE-1 inhibition. Proc. Natl. Acad. Sci. U.S.A. 107, 1630-1635. doi: 10.1073/pnas.0908953107

Johnstone, R. M., Adam, M., Hammond, J. R., Orr, L., and Turbide, C. (1987). Vesicle formation during reticulocyte maturation. Association of plasma membrane activities with released vesicles (exosomes). J. Biol. Chem. 262, 9412-9420.

Jorgensen, M., Baek, R., Pedersen, S., Sondergaard, E. K., Kristensen, S. R., and Varming, K. (2013). Extracellular Vesicle (EV) Array: microarray capturing of exosomes and other extracellular vesicles for multiplexed phenotyping. $J$. Extracell. Vesicles 2:20920. doi: 10.3402/jev.v2i0.20920

Joshi, P., Benussi, L., Furlan, R., Ghidoni, R., and Verderio, C. (2015). Extracellular vesicles in Alzheimer's disease: friends or foes? Focus on $\mathrm{A} \beta$-vesicle interaction. Int. J. Mol. Sci. 16, 4800-4813. doi: 10.3390/ijms 16034800

Jung, S. S., and Levy, E. (2005). Murine cerebrovascular cells as a cell culture model for cerebral amyloid angiopathy: isolation of smooth muscle and endothelial cells from mouse brain. Methods Mol. Biol. 299, 211-219. doi: 10.1007/978-1-61779-551-0_18

Kalra, H., Drummen, G. P., and Mathivanan, S. (2016). Focus on extracellular vesicles: introducing the next small big thing. Int. J. Mol. Sci. 17, 170. doi: 10.3390/ijms17020170

Kowal, J., Tkach, M., and Thery, C. (2014). Biogenesis and secretion of exosomes. Curr. Opin. Cell Biol. 29, 116-125. doi: 10.1016/j.ceb.2014.05.004

Kreimer, S., Belov, A. M., Ghiran, I., Murthy, S. K., Frank, D. A., and Ivanov, A. R. (2015). Mass-spectrometry-based molecular characterization of extracellular vesicles: lipidomics and proteomics. J. Proteome Res. 14, 2367-2384. doi: 10.1021/pr501279t

Lai, R. C., Tan, S. S., Yeo, R. W., Choo, A. B., Reiner, A. T., Su, Y., et al. (2016). MSC secretes at least $3 \mathrm{EV}$ types each with a unique permutation of membrane lipid, protein and RNA. J. Extracell. Vesicles 5:29828. doi: 10.3402/jev.v5.29828

Lakkaraju, A., and Rodriguez-Boulan, E. (2008). Itinerant exosomes: emerging roles in cell and tissue polarity. Trends Cell Biol. 18, 199-209. doi: 10.1016/j.tcb.2008.03.002

Lauritzen, I., Pardossi-Piquard, R., Bauer, C., Brigham, E., Abraham, J. D., Ranaldi, S., et al. (2012). The $\beta$-secretase-derived C-terminal fragment of $\beta A P P, C 99$, but not $A \beta$, is a key contributor to early intraneuronal lesions in triple-transgenic mouse hippocampus. J. Neurosci. 32, 16243-16255. doi: 10.1523/jneurosci.2775-12.2012

Lauritzen, I., Pardossi-Piquard, R., Bourgeois, A., Pagnotta, S., Biferi, M. G., Barkats, M., et al. (2016). Intraneuronal aggregation of the $\beta$-CTF fragment of APP (C99) induces A $\beta$-independent lysosomal-autophagic pathology. Acta Neuropathol. 132, 257-276. doi: 10.1007/s00401-016-1577-6

Lobo, A., Launer, L. J., Fratiglioni, L., Andersen, K., Di Carlo, A., Breteler, M. M., et al. (2000). Prevalence of dementia and major subtypes in Europe: a collaborative study of population-based cohorts. Neurologic Diseases in the Elderly Research Group. Neurology 54(11 Suppl. 5), S4-S9.

McPhie, D. L., Golde, T., Eckman, C. B., Yager, D., Brant, J. B., and Neve, R. L. (2001). $\beta$-secretase cleavage of the amyloid precursor protein mediates neuronal apoptosis caused by familial Alzheimer's disease mutations. Brain Res. Mol. Brain Res. 97, 103-113. doi: 10.1016/S0169-328X(01)00294-7

Mortensen, E. L., and Hogh, P. (2001). A gender difference in the association between APOE genotype and age-related cognitive decline. Neurology 57, 89-95. doi: 10.1212/WNL.57.1.89

Neve, R. L., and Robakis, N. K. (1998). Alzheimer's disease: a re-examination of the amyloid hypothesis. Trends Neurosci. 21, 15-19. doi: 10.1016/S0166-2236 (97)01168-5

Neve, R. L., Boyce, F. M., McPhie, D. L., Greenan, J., and Oster-Granite, M. L. (1996). Transgenic mice expressing APP-C100 in the brain. Neurobiol. Aging 17, 191-203. doi: 10.1016/0197-4580(95)02074-8

Oakley, H., Cole, S. L., Logan, S., Maus, E., Shao, P., Craft, J., et al. (2006). Intraneuronal $\beta$-amyloid aggregates, neurodegeneration, and neuron loss in transgenic mice with five familial Alzheimer's disease mutations: potential factors in amyloid plaque formation. J. Neurosci. 26, 10129-10140. doi: 10.1523/JNEUROSCI.1202-06.2006

Oster-Granite, M. L., McPhie, D. L., Greenan, J., and Neve, R. L. (1996). Agedependent neuronal and synaptic degeneration in mice transgenic for the $\mathrm{C}$ terminus of the amyloid precursor protein. J. Neurosci. 16, 6732-6741.
Ostrowski, M., Carmo, N. B., Krumeich, S., Fanget, I., Raposo, G., Savina, A., et al. (2010). Rab27a and Rab27b control different steps of the exosome secretion pathway. Nat. Cell Biol. 12, 19-30. doi: 10.1038/ ncb2000

Oules, B., Del Prete, D., Greco, B., Zhang, X., Lauritzen, I., Sevalle, J., et al. (2012). Ryanodine receptor blockade reduces amyloid- $\beta$ load and memory impairments in Tg2576 mouse model of Alzheimer disease. J. Neurosci. 32, 11820-11834. doi: 10.1523/JNEUROSCI.0875-12.2012

Patz, S., Trattnig, C., Grunbacher, G., Ebner, B., Gully, C., Novak, A., et al. (2013). More than cell dust: microparticles isolated from cerebrospinal fluid of brain injured patients are messengers carrying mRNAs, miRNAs, and proteins. $J$. Neurotrauma 30, 1232-1242. doi: 10.1089/neu.2012.2596

Payami, H., Montee, K. R., Kaye, J. A., Bird, T. D., Yu, C. E., Wijsman, E. M., et al. (1994). Alzheimer's disease, apolipoprotein E4, and gender. JAMA 271, 1316-1317. doi: 10.1001/jama.1994.03510410028015

Perez-Gonzalez, R., Gauthier, S. A., Kumar, A., and Levy, E. (2012). The exosomesecretory pathway transports amyloid precursor protein carboxyl terminal fragments from the cell into the brain extracellular space. J. Biol. Chem. 287, 43108-43115. doi: 10.1074/jbc.M112.404467

Pfeffer, S. R. (2013). Rab GTPase regulation of membrane identity. Curr. Opin. Cell Biol. 25, 414-419. doi: 10.1016/j.ceb.2013.04.002

Pisitkun, T., Shen, R. F., and Knepper, M. A. (2004). Identification and proteomic profiling of exosomes in human urine. Proc. Natl. Acad. Sci. U.S.A. 101, 13368-13373. doi: 10.1073/pnas.0403453101

Pituch, K. C., Moyano, A. L., Lopez-Rosas, A., Marottoli, F. M., Li, G., Hu, C., et al. (2015). Dysfunction of platelet-derived growth factor receptor $\alpha$ (PDGFR $\alpha$ ) represses the production of oligodendrocytes from arylsulfatase Adeficient multipotential neural precursor cells. J. Biol. Chem. 290, 7040-7053. doi: 10.1074/jbc.M115.636498

Polanco, J. C., Scicluna, B. J., Hill, A. F., and Gotz, J. (2016). Extracellular vesicles isolated from brains of rTg4510 mice seed tau aggregation in a threshold-dependent manner. J. Biol. Chem. 291, 12445-12466. doi: 10.1074/ jbc.M115.709485

Pols, M. S., and Klumperman, J. (2009). Trafficking and function of the tetraspanin CD63. Exp. Cell Res. 315, 1584-1592. doi: 10.1016/j.yexcr.2008.09.020

Rajendran, L., Honsho, M., Zahn, T. R., Keller, P., Geiger, K. D., Verkade, P., et al. (2006). Alzheimer's disease $\beta$-amyloid peptides are released in association with exosomes. Proc. Natl. Acad. Sci. U.S.A. 103, 11172-11177. doi: 10.1073/pnas.0603838103

Record, M., Subra, C., Silvente-Poirot, S., and Poirot, M. (2011). Exosomes as intercellular signalosomes and pharmacological effectors. Biochem. Pharmacol. 81, 1171-1182. doi: 10.1016/j.bcp.2011.02.011

Saman, S., Kim, W., Raya, M., Visnick, Y., Miro, S., Jackson, B., et al. (2012). Exosome-associated tau is secreted in tauopathy models and is selectively phosphorylated in cerebrospinal fluid in early Alzheimer disease. J. Biol. Chem. 287, 3842-3849. doi: 10.1074/jbc.M111.277061

Selkoe, D. J. (1991). The molecular pathology of Alzheimer's disease. Neuron 6, 487-498. doi: 10.1016/0896-6273(91)90052-2

Sharples, R. A., Vella, L. J., Nisbet, R. M., Naylor, R., Perez, K., Barnham, K. J., et al. (2008). Inhibition of $\gamma$-secretase causes increased secretion of amyloid precursor protein $\mathrm{C}$-terminal fragments in association with exosomes. FASEB J. 22, 1469-1478. doi: 10.1096/fj.07-9357com

Simpson, R. J., Jensen, S. S., and Lim, J. W. (2008). Proteomic profiling of exosomes: current perspectives. Proteomics 8, 4083-4099. doi: 10.1002/pmic. 200800109

Smalheiser, N. R. (2007). Exosomal transfer of proteins and RNAs at synapses in the nervous system. Biol. Direct 2:35. doi: 10.1186/1745-6150-2-35

Spencer, B., Kim, C., Gonzalez, T., Bisquertt, A., Patrick, C., Rockenstein, E., et al. (2016). $\alpha$-synuclein interferes with the ESCRT-III complex contributing to the pathogenesis of Lewy body disease. Hum. Mol. Genet. 25, 1100-1115. doi: $10.1093 / \mathrm{hmg} / \mathrm{ddv} 633$

Stine, W. B. Jr., Dahlgren, K. N., Krafft, G. A., and LaDu, M. J. (2003). in vitro characterization of conditions for amyloid- $\beta$ peptide oligomerization and fibrillogenesis. J. Biol. Chem. 278, 11612-11622. doi: 10.1074/jbc.M210 207200

Stoorvogel, W., Kleijmeer, M. J., Geuze, H. J., and Raposo, G. (2002). The biogenesis and functions of exosomes. Traffic 3, 321-330. doi: $10.1034 / j .1600-0854.2002 .30502 . x$ 
Tamayev, R., and D'Adamio, L. (2012). Inhibition of $\gamma$-secretase worsens memory deficits in a genetically congruous mouse model of Danish dementia. Mol. Neurodegener. 7:19. doi: 10.1186/1750-1326-7-19

Tamayev, R., Matsuda, S., Arancio, O., and D'Adamio, L. (2012). $\beta$-but not $\gamma$-secretase proteolysis of APP causes synaptic and memory deficits in a mouse model of dementia. EMBO Mol. Med. 4, 171-179. doi: 10.1002/emmm.201100195

Thery, C., Amigorena, S., Raposo, G., and Clayton, A. (2006). Isolation and characterization of exosomes from cell culture supernatants and biological fluids. Curr. Protoc. Cell Biol. Chapter 3:Unit 3.22. doi: 10.1002/0471143 030.cb0322s30

Urbanelli, L., Magini, A., Buratta, S., Brozzi, A., Sagini, K., Polchi, A., et al. (2013). Signaling pathways in exosomes biogenesis, secretion and fate. Genes 4, 152-170. doi: 10.3390/genes4020152

van der Pol, E., Boing, A. N., Gool, E. L., and Nieuwland, R. (2015). Recent developments on the nomenclature, presence, isolation, detection and clinical impact of extracellular vesicles. J. Thromb. Haemost. 14, 48-56. doi: $10.1111 /$ jth. 13190

van der Pol, E., Boing, A. N., Harrison, P., Sturk, A., and Nieuwland, R. (2012). Classification, functions, and clinical relevance of extracellular vesicles. Pharmacol. Rev. 64, 676-705. doi: 10.1124/pr.112.005983

Vella, L. J., Hill, A. F., and Cheng, L. (2016). Focus on extracellular vesicles: exosomes and their role in protein trafficking and biomarker potential in Alzheimer's and Parkinson's disease. Int. J. Mol. Sci. 17, 173. doi: 10.3390/ijms17020173

Vella, L. J., Sharples, R. A., Nisbet, R. M., Cappai, R., and Hill, A. F. (2008). The role of exosomes in the processing of proteins associated with neurodegenerative diseases. Eur. Biophys. J. 37, 323-332. doi: 10.1007/s00249-0070246-Z

Vingtdeux, V., Hamdane, M., Loyens, A., Gele, P., Drobeck, H., Begard, S., et al. (2007). Alkalizing drugs induce accumulation of amyloid precursor protein by-products in luminal vesicles of multivesicular bodies. J. Biol. Chem. 282, 18197-18205. doi: 10.1074/jbc.M609475200

Willms, E., Johansson, H. J., Mager, I., Lee, Y., Blomberg, K. E., Sadik, M., et al. (2016). Cells release subpopulations of exosomes with distinct molecular and biological properties. Sci. Rep. 6:22519. doi: 10.1038/srep22519

Yelamanchili, S. V., Lamberty, B. G., Rennard, D. A., Morsey, B. M., Hochfelder, C. G., Meays, B. M., et al. (2015a). MiR-21 in extracellular vesicles leads to neurotoxicity via TLR7 signaling in SIV neurological disease. PLoS Pathog. 11:e1005032. doi: 10.1371/journal.ppat.1005032

Yelamanchili, S. V., Lamberty, B. G., Rennard, D. A., Morsey, B. M., Hochfelder, C. G., Meays, B. M., et al. (2015b). Correction: MiR-21 in extracellular Vesicles leads to neurotoxicity via TLR7 signaling in SIV neurological disease. PLoS Pathog. 11:e1005131. doi: 10.1371/journal.ppat.1005131

Yuyama, K., Sun, H., Mitsutake, S., and Igarashi, Y. (2012). Sphingolipidmodulated exosome secretion promotes the clearance of amyloid- $\beta$ by microglia. J. Biol. Chem. 287, 10977-10989. doi: 10.1074/jbc.M111.324616

Zhou, H., Yuen, P. S., Pisitkun, T., Gonzales, P. A., Yasuda, H., Dear, J. W., et al. (2006). Collection, storage, preservation, and normalization of human urinary exosomes for biomarker discovery. Kidney Int. 69, 1471-1476. doi: $10.1038 /$ sj.ki.5000273

Conflict of Interest Statement: The author declares that the research was conducted in the absence of any commercial or financial relationships that could be construed as a potential conflict of interest.

Copyright (C) 2017 Levy. This is an open-access article distributed under the terms of the Creative Commons Attribution License (CC BY). The use, distribution or reproduction in other forums is permitted, provided the original author (s) or licensor are credited and that the original publication in this journal is cited, in accordance with accepted academic practice. No use, distribution or reproduction is permitted which does not comply with these terms. 